\section{Qwiffs and quantum leaps}

\author{
Brian D. Josephson
}

Mind and the New Physics*. By Fred Alan Wolf. Heinemann:1985. Pp.342. £14.95.

IN THIS book the author expounds his ideas relating to a postulated connection between quantum mechanics and mind. a connection which is now beginning to be recognized as being possibly important by a number of scientists. Wolf starts from the conventional point of view that the quantum-mechanical wave function (or "qwiff" in his terminology) represents the potential for anything physical to become manifest. and regards the process of making decisions as one of interaction with the (potential) future. He postulates that we have control over the future by deciding what to observe. and thus determining which way the wave function should collapse. We capture possibilities by observing them and collapsing the wave function appropriately in the same way that a fisherman captures fish in his net. The author additionally discusses in a rather metaphorical way some informationprocessing aspects of quantum theory, as well as a large number of other, on the whole rather more questionable, ideas.

While the putative mechanism for being able to control the future has a certain degree of plausibility, conventional quantum theory would argue that the chance of not finding a particular possibility of interest should be taken into account as well as the one of actually finding it, these two possible outcomes being associated with wave function collapses in different directions. This would seem to lead to theoretical predictions distinctly different from those proposed by Wolf. His process for modifying the outcome would work only if a differential collapse mechanism were in operation, and would make the theory very close to E.H. Walker's theory of the control of physical reality by the will which was published in Vol. 3 of Psychoenergetic Systems (1979).

Unfortunately, the kind of attention to detail and use of logical methods of argument that might have made the author aware of matters such as the discrepancy between the mechanism he proposes and conventional theory is not a feature of this book. Its basic structure is one of informal expositions of standard theory, followed up by intuitions about their meaning in the author's scheme of things. Despite my sympathy with what Wolf is trying to do, it seems to me that the connections proposed, while occasionally being significant, have been arrived at mainly by a process of coupling together any two en-

\footnotetext{
* In the United States Star Wave published by Macmillan, New York: Hbk \$19.95, pbk \$7.95.
}

tities that have some nebulous resemblance to each other (a typical example is the connection between Bose-Einstein statistics and love. discussed on pp.152154)

One can have little more confidence in the mathematical parts of the book. the illustrations for which have been produced using a computer apparently lacking graphics facilities. which hardly makes them easier to follow. Starting with an elementary howler in the example given to illustrate the addition of two wave functions on p.191. which might be excused as a momentary lapse of attention. the author goes on to describe the equation $\langle$ l/I $\rangle=1$ as representing the law "The Mind is One". He then proceeds further to equations supposedly representing the characteristics of Enterprise. God and Satan, at which point the book starts to bear a distinct resemblance to some of the more dubious offerings from the public that arrive occasionally in one's mail box. It could be that there is some sense behind these strings of equations, but unfortunately Wolf does not consider it important to go into the details so that the critical reader can judge for himself.

\section{Taking an aim}

\section{Gregory Gregoriadis}

Red Blood Cells as Carriers for Drugs. Edited by J.R. DeLoach and U. Sprandel. Karger: 1985. Pp.162. DM 125, \$44.25.

THE development of carriers to obtain optimal release of drugs where they are needed, and protection from hostile environments or premature loss, is a subject of growing interest and a hopeful bet in biotechnology ventures. Currently popular carriers include antibodies, polymers, liposomes and other colloids. With only a minority of workers favouring erythrocytes, what then is so special about them to warrant a meeting and a book? Several things according to the editors and the contributors. Most medical researchers are familiar with erythrocytes, a known quantity to be trusted, by clinicians especially (the danger of unwanted contaminants in other people's blood, is avoided by using the patient's own erythrocytes).

Erythrocytes can, in theory at least, optimize drug action in more than one way. For instance, they end up sooner or later in the reticuloendothelial system which is associated with a large number of microbial and other diseases, and could therefore carry drugs there. This aspect is discussed in chapters on the use of cells loaded with metal chelators which facilitate removal of stored iron, or with hydrolytic enzymes which can break down stored substrates in the tissues of patients with storage diseases. Further, (human) erythrocytes have a life span of over 100 days, an important feature for slow re-
The side effect of books such as this maly be to cause other books and journal articles, which treat this subject in a more serious manner. to miss the attention they deserve. There come to mind Rupert Sheldrake's $A$ New Science of Life, which has been criticized mainly on account of its unorthodoxy and not because of any errors or inconsistencies in its arguments: Fritjof Capra's Tao of Physics; D. Bohm and B.J. Hiley's article ( $F$ dn Phys. USA 14, 255-274: 1984) which indicated that mind-like characteristics of nature may be inherent in the equations of quantum physics; and the two papers of H.P. Stapp (Fdn Phys. USA 12, 363-399; 1982 and $15,35-47 ; 1985)$ on the connection between mind. matter and quantum mechanics. On the assumption that important new ideas may in fact be buried in Wolf's book. one must hope that a subsequent, more rigorous account will be written. directed at the scientist rather than at the non-scientist who seems to be the target of this one.

Brian D. Josephson is Professor of Phvsics in the Cavendish Laboratory. Universiny of Cam bridge. Madingley Road. Cambridge ( $B 30 \mathrm{HE}$ $U K$.

lease of drugs into the circulation.

The book describes experiences with the release of antimicrobial and anticancer drugs from circulating erythrocytes. Inactivation of toxic metabolites entering erythrocytes by enzymes which have been introduced into the cells is discussed in chapters on asparaginase, rhodanese (coentrapped with sodium thiosulphate for cyanide detoxification), or urate oxidasecontaining erythrocytes. Other applications include erythrocyte-mediated introduction of DNA and antibodies into eukaryotic cells and the enrichment of erythrocytes with allosteric effectors of haemoglobin or with 6-phosphate dehydrogenase.

Is this all too good to be true? Unfortunately yes. The editors and some of the authors recognize the formidable problems with the proposed uses, especially in terms of wide acceptability. For instance. there are already a number of simple and safe man-made carriers which can deliver drugs to the reticuloendothelial system efficiently. Further, release of drugs from circulating cells at a constant rate will probably be impossible to achieve. Perhaps the greatest advantage of erythrocytes over other systems is their long circulation time and their use in detoxification looks realistic enough. The book, discussing most aspects of the erythrocyte carrier and related technology in 19 short chapters, is the first one devoted entirely to this system and should prove useful to those interested in drug delivery.

Gregory (iregoriadis is Head. Medical Research Council Group. Academic Deparment of Medicine. Roval Free Hospital School of Medicine, London NW32QG. UK 